\title{
Inercia clínica en el tratamiento con insulina en el primer nivel de atención
}

\author{
Felipe Vázquez, ${ }^{1}$ Pilar Lavielle, ${ }^{2}$ Rita Gómez-Díaz ${ }^{2}$ y Niels Wacher ${ }^{2}$
}

${ }^{1}$ Secretaría de Salud, Servicios de Atención Psiquiátrica; ${ }^{2}$ Instituto Mexicano del Seguro Social, Centro Médico Nacional Siglo XXI, Hospital de Especialidades, Unidad de Investigación en Epidemiología Clínica. Ciudad de México, México

\begin{abstract}
Resumen
Introducción: Poco se ha evaluado el rechazo de los médicos a prescribir insulina a sus pacientes; el retraso en intensificar el tratamiento impide una atención adecuada y de calidad. Objetivo: Identificar la percepción de los médicos acerca de las barreras para iniciar la insulina en los pacientes con diabetes. Método: Por Índice Smith y análisis multivariado, en 81 médicos familiares se evaluó la relevancia y agrupación de los conceptos relacionados con las barreras para la prescripción de insulina. Resultados: 35.8 \% de los médicos mostró confianza en prescribir insulina; casi la mitad calificó la intensificación del tratamiento entre moderadamente y poco importante (39.5 y $6.2 \%$ ). Las barreras se relacionaron con el médico (39.5\%), el paciente (37\%), el tratamiento con insulina (11.1\%) y la institución (6.2\%); $6.2 \%$ de los médicos no percibió ninguna barrera. Las barreras se agruparon en cinco factores, que explicaron $62.48 \%$ de la varianza: cultura de los pacientes, falta de habilidades, miedo a los eventos adversos, inseguridad y falta de capacitación. Conclusión: La inercia clínica no resultó de una condición clínica compleja o comorbilidades del paciente, sino de la percepción del médico y de su confianza en sus habilidades clínicas y comunicativas.
\end{abstract}

PALABRAS CLAVE: Inercia clínica. Atención primaria. Tratamiento con insulina. Barreras.

\begin{abstract}
Introduction: Refusal of physicians to prescribe insulin to their patients has been scarcely evaluated; the delay in treatment intensification hinders adequate and quality care. Objective: To identify the perception of primary care physicians about barriers to initiate insulin treatment in patients with diabetes. Method: Using the Smith Index and multivariate analysis, the relevance and grouping of concepts related to barriers to insulin prescription were assessed in 81 family doctors. Results: Only 35.8\% of physicians showed confidence for prescribing insulin; almost half of them rated treatment intensification between moderately and little important (39.5\% and 6.2\%). Barriers were related to the physician (39.5\%), the patient (37\%), insulin treatment (11.1\%) and the institution (6.2\%); $6.2 \%$ of physicians did not perceive any barrier. The barriers were grouped in 5 factors that explained $62.48 \%$ of the variance: patient cultural level, lack of medical skills, fear of adverse events, insecurity and lack of training. Conclusion: Clinical inertia was not the result of a complex medical condition or patient comorbidities, but of doctor's perception and confidence in his/her clinical and communication skills.
\end{abstract}

KEY WORDS: Clinical inertia. Primary care. Insulin treatment. Barriers.

Correspondencia:

Pilar Lavielle

E-mail: lavielle.pilar@gmail.com
Fecha de recepción: 16-08-2018

Fecha de aceptación: 05-01-2019

DOI:10.24875/GMM.19004582
Gac Med Mex. 2019;155:156-161

Disponible en PubMed www.gacetamedicademexico.com 


\section{Introducción}

La prevención y tratamiento de la diabetes tipo 2 (DM2) se ha convertido en uno de los principales retos para las instituciones de salud. La prevalencia global de este padecimiento se ha incrementado de $4.7 \%$ en 1980 a $8.5 \%$ en $2014 .{ }^{1}$ En Europa se ha estimado que la prevalencia de la diabetes es de $7.8 \%$, en Norteamérica y el Caribe de $11.4 \%$ y en Sudamérica, de $8.1 \%{ }^{2}$ En México, los resultados de la Encuesta $\mathrm{Na}$ cional de Salud y Nutrición de medio camino (ENSANUT 2016) mostraron que la prevalencia de casos conocidos de diabetes fue de $9.2 \%{ }^{3}$ Esta encuesta también reportó que únicamente $25.3 \%$ de los pacientes alcanzaba los objetivos terapéuticos de hemoglobina glucosilada (HbA1c) $<7 \%$ y solo $13 \%$ de quienes recibían atención médica fueron tratados con insulina para el control de la hiperglucemia. ${ }^{4}$

Numerosos pacientes con diabetes tratados inicialmente con dieta, ejercicio o medicamentos eventualmente requerirán la intensificación del tratamiento. ${ }^{5}$ No obstante, se ha demostrado que esta medida terapéutica se puede retrasar por varios años, particularmente cuando la insulina es la siguiente opción terapéutica. ${ }^{6}$ Diversos estudios se han enfocado en la "resistencia psicológica a la insulina" de los pacientes, término que refiere la negativa de estos a aceptar el tratamiento con insulina debido a expectativas negativas sobre los resultados, ${ }^{7,8}$ sin embargo, pocos evalúan el rechazo de los médicos a prescribir insulina. ${ }^{9}$ Este fenómeno -conocido como "inercia clínica" - se caracteriza por la incapacidad del médico para intensificar el tratamiento oportunamente, retraso que constituye uno de los factores más importantes que impiden la atención adecuada y de calidad a los pacientes con DM2 (es decir, corregir el control glucémico deficiente). ${ }^{10}$ De acuerdo con los estudios publicados, la inercia clínica puede afectar entre $30 \mathrm{y}$ $68 \%$ de los casos, proporción que puede incrementarse cuando la $\mathrm{HbA} 1 \mathrm{c}$ es mayor de $9 \%{ }^{11}$

Dado que la inercia clínica es principalmente responsabilidad del médico ${ }^{12}$ y que el cambio de comportamiento requiere que el individuo perciba su importancia y se sienta seguro sobre lo que debe hacer (autoeficacia), el presente estudio tuvo como objetivo identificar la percepción del médico familiar acerca de la importancia, barreras y confianza para iniciar el tratamiento con insulina en pacientes con diagnóstico de DM2. Este conocimiento puede ser de gran ayuda para implementar intervenciones apropiadas para apoyar al médico familiar a utilizar adecuadamente el tratamiento con insulina y como consecuencia, incrementar la proporción de pacientes que logren los objetivos terapéuticos respecto a los niveles de HbA1c.

\section{Método}

Para asegurar la validez de los datos se llevó a cabo un estudio mixto (encuestas cuantitativas que se combinaron con métodos cualitativos).

Fue invitada a participar una muestra propositiva por conveniencia de 81 médicos familiares que asistieron al Centro de Capacitación del Instituto Mexicano del Seguro Social para participar en un curso sobre DM2 dirigido a incrementar la calidad de la atención en este padecimiento. El curso formó parte del programa DiabetIMSS y del protocolo aprobado por el Comité de Investigación de la institución (IMSS2004-131). Los médicos dieron su consentimiento verbal para participar después de la explicación de los objetivos y dinámica del estudio.

Se llevaron a cabo entrevistas semiestructuradas para conocer la percepción de los médicos acerca de las barreras para iniciar el tratamiento con insulina..$^{13}$ En una tarjeta, los médicos contestaron una pregunta (de forma anónima) que evaluaba la percepción acerca de las barreras a las que se enfrentaban en la práctica clínica diaria para iniciar el tratamiento con insulina a los pacientes con DM2. Por medio de una escala visual análoga de 10 puntos (en la que el 0 fue la valoración mínima), los médicos calificaron el grado de importancia y confianza que experimentaban en la prescripción de insulina.

Las entrevistas fueron evaluadas mediante análisis de contenido o codificación (proceso de reducción de la información cualitativa) para obtener datos cualitativos y cuantitativos. El análisis de contenido es una metodología de investigación basada en procedimientos estandarizados que se enfoca en el estudio de los contenidos y su objetivo es la descripción sistemática, válida y reproducible de la comunicación o cualquier otra manifestación de la conducta. El significado de las palabras, temas o frases es lo que se cuantifica.

Con base a esta técnica, los conceptos extraídos de las entrevistas fueron clasificados en cierto número de categorías de acuerdo con su similitud semántica, que reflejaban los patrones cognitivos del grupo estudiado. Este procedimiento permitió construir una matriz de dos vías (participante/categoría) y realizar un análisis cuantitativo para calcular las frecuencias 
de los conceptos mencionados por los participantes en las entrevistas. ${ }^{14}$

Posteriormente se calculó el Índice Smith o salience score para determinar qué categoría sobre las barreras en la prescripción de insulina era la más relevante. El cálculo del índice de preponderancia es una técnica de la antropología cognitiva -utilizada en múltiples disciplinas científicas- para describir y analizar la cultura y las conductas de los participantes desde su punto de vista. Este índice se calcula a partir de dos criterios:

- Los conceptos mencionados primero.

- Los conceptos mencionados con mayor frecuencia.

Con base en este índice se describe lo que las personas piensan respecto a un fenómeno determinado y la forma como organizan esta información, para determinar los conceptos más importantes para los entrevistados en relación con el tema investigado, en este caso las barreras para prescribir insulina. ${ }^{15,16}$ Las ventajas más importantes de esta técnica es que produce información profunda del proceso cognitivo de los sujetos a través de un método fácil de aplicar y de entender por los participantes. Se incluyeron solo los conceptos mencionados con una frecuencia $\geq 4$, debido a que los conceptos referidos con menor frecuencia no eran compartidos por el grupo. ${ }^{17}$

Finalmente, de acuerdo con los objetivos del estudio y el tipo de datos generados, se llevó a cabo un análisis multivariado de componentes principales para describir la relación entre los conceptos, ${ }^{18,19}$ examinando aquellos que se agruparon en el mismo factor (según los criterios de Kaiser, se conservaron los factores cuyo autovalor asociado fue mayor que 1).

\section{Resultados}

De 81 médicos familiares entrevistados, $22.7 \%$ fue del sexo femenino. La media de edad fue de 45 años. El promedio de años de experiencia como médico familiar fue de 15 años.

La mayoría de los médicos participantes reportó bajos niveles de confianza en sus habilidades para iniciar el tratamiento con insulina a los pacientes con diabetes: $14.8 \%$ reconoció no sentir confianza en sus habilidades, $49.4 \%$ se sentía moderadamente confiado y solo $35.8 \%$ se mostró confiado en proporcionar este tipo de tratamiento. Un hallazgo sorprendente fue que casi la mitad de los médicos familiares calificó la intensificación del tratamiento a los pacientes con diabetes entre moderada y poco importante (39.5 y $6.2 \%$, respectivamente).
Tabla 1. Principales categorías mencionadas por los médicos familiares acerca de las barreras para prescribir insulina, según el Índice Smith (orden y frecuencia de mención)

\begin{tabular}{lccc}
\hline Categoría & $\mathbf{n}$ & $\begin{array}{c}\% \text { de } \\
\text { respuesta* }\end{array}$ & $\begin{array}{c}\text { Índice } \\
\text { Smith }\end{array}$ \\
\hline $\begin{array}{l}\text { Limitaciones culturales del } \\
\text { paciente }\end{array}$ & 16 & 19.75 & 0.28 \\
Rechazo del paciente & 12 & 14.81 & 0.27 \\
Hipoglucemia & 12 & 14.81 & 0.25 \\
Capacitación del médico & 14 & 17.28 & 0.18 \\
Adherencia del paciente & 9 & 11.11 & 0.18 \\
Inexperiencia médica & 8 & 9.87 & 0.18 \\
Falta de habilidades de & 6 & 7.40 & 0.1 \\
comunicación del médico & & & 0.1 \\
Inseguridad del médico & 5 & 6.17 & 0.08 \\
\hline $\begin{array}{l}\text { Ignorancia del paciente } \\
\text { *Porcentaje de médicos que respondieron a la categoría indicada. }\end{array}$
\end{tabular}

El análisis de las entrevistas mostró que las barreas para iniciar el tratamiento con insulina se relacionaron con características del médico en $39.5 \%$, el paciente en $37 \%$ y el tratamiento en $11.1 \%$. Solo $6.2 \%$ de los médicos familiares no percibía ninguna barrera para iniciar insulina a sus pacientes y comentó que ya la prescribía en quienes la requerían, con resultados positivos. Finalmente, $6.2 \%$ consideró que la institución también era una barrera en la prescripción.

De acuerdo con el Índice Smith (Tabla 1), las principales barreras que perciben los médicos familiares para prescribir insulina fueron la cultura de los pacientes y la falta de capacitación de los médicos, seguidas por el rechazo del paciente para recibir el tratamiento y los efectos adversos de la insulina, como la hipoglucemia.

Con las nueve categorías compartidas por los médicos acerca de las barreras para la prescripción de insulina se llevó a cabo un análisis multivariado de componentes principales, para determinar la relación entre ellas. De acuerdo con el análisis, las categorías se agruparon en cinco factores (que alcanzaron valores-eigen mayores de 1 de acuerdo con el criterio Kaiser), como se muestra en la Tabla 2. Estos cinco factores explicaron $62.48 \%$ de la varianza: factor $1,15.4 \%$; factor $2,13.3 \%$; factor 3 , $12.3 \%$; factor 4, $10.9 \%$; factor $5,10.4 \%$ :

\section{Factor I}

Este factor estuvo integrado por los aspectos culturales y cognitivos de los pacientes que los médicos 
Tabla 2. Agrupación de los conceptos mencionados por los médicos familiares acerca de las barreras para prescribir insulina, según el análisis de factores

\begin{tabular}{lccccc}
\hline Concepto & $\begin{array}{c}\text { Factor } \\
\text { I }\end{array}$ & $\begin{array}{c}\text { Factor } \\
\text { II }\end{array}$ & $\begin{array}{c}\text { Factor } \\
\text { III }\end{array}$ & $\begin{array}{c}\text { Factor } \\
\text { IV }\end{array}$ & $\begin{array}{c}\text { Factor } \\
\text { V }\end{array}$ \\
\hline Ignorancia del paciente & 0.648 & & & \\
Cultura del paciente & 0.624 & & & \\
Inexperiencia médica & & 0.407 & & \\
Falta de habilidades & & 0.534 & & \\
de comunicación del & & & & \\
médico & & & & \\
Rechazo del paciente & 0.480 & & \\
Hipoglucemia & & & 0.643 & \\
No adherencia & & & 0.285 & & \\
Inseguridad del médico & & & & 0.859 & \\
Capacitación médica & & & & 0.310 \\
\hline Método de extracción,análisis de componentes principales. &
\end{tabular}

consideraron como las barreras más importantes para la prescripción de la insulina. Los principales conceptos mencionados fueron "mitos que tienen los pacientes sobre la insulina", "tabús de los pacientes", "la cultura de los pacientes que no les permite aceptar el tratamiento", "estigma", "poca aceptación de los pacientes de la insulina", "creencias de los pacientes de que la insulina puede dejarlos ciegos".

\section{Factor II}

En este factor están integradas las actitudes y conductas negativas de los médicos que incrementan el rechazo de los pacientes hacia el tratamiento con insulina. Incluye las barreras reportadas por los médicos acerca de su falta de experiencia en el manejo de la insulina, así como la deficiencia en las habilidades de comunicación que no les ha permitido presentar adecuadamente al paciente el balance costo-beneficio del tratamiento con insulina: "no tengo suficiente experiencia", "no lo he hecho antes", "soy incapaz de convencer al paciente", "no tengo la capacidad de presentar la información adecuada para convencer al paciente", "debemos incrementar la conciencia y confianza del paciente".

\section{Factor III}

Los médicos reportaron que una de las barreras para la prescripción de insulina es el miedo a los efectos adversos del medicamento (hipoglucemia). En este factor sobresalen los propios miedos del médico, desde causar hipoglucemia o ser criticados por otros médicos, hasta el temor a causar la muerte al paciente: "aplicar una dosis que pudiera producir hipoglucemia", "como nunca la he usado, tengo miedo de que el paciente sufra de hipoglucemia y nadie se dé cuenta".

\section{Factor IV}

Los médicos reportaron sus sentimientos de inseguridad en relación con el manejo de la insulina: "inseguridad", "falta de confianza en el manejo", "falta de confianza en prescribir insulina nocturna". Algunos médicos no están motivados a prescribir insulina y preferían referir al paciente con el endocrinólogo.

\section{Factor $V$}

En este factor se incluyó la deficiente capacitación de los médicos para el manejo de la insulina y para identificar a los candidatos adecuados para recibirla: "falta de capacitación", "aprender quiénes son los pacientes correctos para el tratamiento de insulina y cómo usarla".

\section{Barreas relacionadas con la institución de salud}

La institución de salud no fue uno de los aspectos que se reportaron con frecuencia, sin embargo, es pertinente mencionar que algunos médicos mencionaron las dificultades para obtener la insulina en la farmacia y la relación profesional inadecuada con otros especialistas en los hospitales generales

\section{Discusión}

El presente estudio proporciona información importante sobre una amplia gama de creencias y actitudes de los médicos acerca de intensificar el tratamiento en los pacientes con DM2. Nuestro estudio se basó en entrevistas semiestructuradas con médicos de atención primaria que participaron en un curso sobre diabetes para mejorar la calidad de la atención. Este tipo de muestra y enfoque metodológico permitió $-a$ través de las reflexiones de los médicos- conocer los sentimientos y creencias personales sobre la experiencia con la prescripción de la insulina y comprender el fenómeno de la inercia clínica. 
Identificamos que las cinco principales causas de la inercia clínica percibidas por los médicos fueron antecedentes culturales de los pacientes, habilidades médicas deficientes, miedo a los eventos adversos relacionados con la insulina, inseguridad médica y falta de capacitación.

Para los médicos de nuestro estudio, el principal factor que explica la inercia clínica fue el rechazo de los pacientes al tratamiento con insulina, basado en creencias culturales como tabús y mitos acerca de la insulina, sentirse estigmatizados, así como en procesos cognitivos que generaban atribuciones negativas y factores emocionales, como el miedo. No obstante, esta apreciación no es exacta. En otras investigaciones se ha reportado que los médicos estiman a priori la probabilidad del rechazo de los pacientes a la intensificación del tratamiento, sin haberlos consultado antes de tomar una decisión, por ello no queda claro qué tan precisas son las estimaciones de los médicos acerca de las preferencias o las capacidades de automanejo de los pacientes. ${ }^{20}$ Por ejemplo, se ha estimado que $30 \%$ de los pacientes que no se adhieren al tratamiento señalaron que tenían miedo a las inyecciones, mientras que $97 \%$ de los médicos consideró que esta era la principal causa de la falta de adherencia. ${ }^{21} \mathrm{En}$ contraste, médicos de otras especialidades (endocrinología o medicina interna) no piensan que esta sea una barrera para el tratamiento con insulina. La diferencia en la percepción de los médicos acerca de las barreras de los pacientes puede reflejar la resistencia de los médicos familiares para prescribir insulina. ${ }^{20,22}$

Estos datos se relacionan con otro hallazgo: la prescripción de insulina incrementa el estrés en el médico familiar (poco explorado en estudios previos sobre inercia clínica). Los participantes de este estudio reconocieron sentimientos de inseguridad, falta de experiencia en la prescripción de insulina y miedo a los posibles efectos adversos. ${ }^{23,24}$ Estos sentimientos negativos se relacionaron con la percepción de que la deficiente capacitación y habilidades de comunicación son el principal impedimento para presentar un balance adecuado costo-beneficio, lo cual a su vez lleva a que el paciente rechace iniciar el tratamiento con insulina. Estos resultados son acordes con los de otros estudios en los que se encontró que la prescripción de insulina produce altos niveles de ansiedad en los médicos familiares, cuyas emociones pueden ser una de las principales razones para la inercia clínica.
Diversas investigaciones han mostrado las consecuencias negativas que estos factores emocionales pueden producir. Por un lado, una conducta demasiado cautelosa en la práctica clínica, que reduce el acceso de los pacientes a procedimientos específicos (como la prescripción de insulina), afectando la calidad de la atención médica. ${ }^{25}$ Por otro lado, al percibir la inseguridad del médico, los pacientes pueden considerar la prescripción de insulina como una decisión médica incorrecta, especialmente si perciben actitudes negativas en los médicos, como la amenaza de iniciar el tratamiento con insulina como un castigo ante la falta de adherencia. ${ }^{26}$ Estos factores pueden desempeñar un papel muy importante en la aceptación del paciente del tratamiento con insulina y convertirse en una barrera en el control metabólico. ${ }^{27}$

Como lo sugieren los resultados de nuestra investigación, es importante incrementar la confianza y seguridad del médico familiar en sus habilidades clínicas para la prescripción de insulina a los pacientes con DM2, a través de programas de capacitación en esta área, que también incluyan estrategias psicológicas y de comunicación. Lo anterior puede influir positivamente en la forma de comprender y recordar las instrucciones del médico, en los resultados clínicos del paciente y en la calidad de la atención. ${ }^{28,29}$

Este estudio tiene algunas limitaciones que es importante tener en cuenta. La muestra utilizada podría llevar a una sobreestimación de los resultados: debido a que los médicos entrevistados estaban participando en un curso para mejorar la atención del paciente con diabetes, es posible que percibieran algunas deficiencias en esta área, apreciación que pudiera no ser compartida por otros médicos familiares. Por lo tanto, es importante realizar un estudio con una muestra representativa de médicos de familiares para corroborar los resultados.

\section{Conclusiones}

Independientemente de las consideraciones del médico sobre la importancia del tratamiento con insulina, la inercia clínica no fue resultado de una condición clínica compleja o comorbilidades del paciente, sino de sentimientos y creencias de los médicos familiares acerca de la conducta del paciente y la propia confianza en sus habilidades clínicas y de comunicación, lo cual afecta la calidad de la atención que proporcionan. 


\section{Agradecimientos}

Los autores agradecen a Susan Drier Jonas, por su apoyo con el manuscrito.

\section{Bibliografía}

1. World Health Organization. Global status report on non-communicable diseases. Ginebra, Suiza: World Health Organization; 2014.

2. International Diabetes Federation. IDF Diabetes Atlas. Octava edición. Bélgica: International Diabetes Federation; 2018.

3. Hernández-Ávila M, Rivera-Dommarco J, Shamah-Levy T, Cuevas-Nasu L, Gómez-Acosta LM, Gaona-Pineda EB et al. Encuesta Nacional de Salud y Nutrición de Medio Camino 2016. Informe final de resultados. México: Secretaría de Salud/Instituto Nacional de Salud Pública; 2016.

4. Hernández-Ávila M, Gutiérrez JP, Reynoso-Noverón N. Diabetes mellitus in Mexico. Status of the epidemic. Salud Publica Mex. 2013;55:S129-S136.

5. Barag SH. Insulin therapy for management of type 2 diabetes mellitus: strategies for initiation and long-term patient adherence. J Am Osteopath Assoc. 2011;111:S13-S19.

6. Brath H, Paldánius P, Bader G, Kolaczynski WM, Nilsson P. Differences in glycemic control across world regions: a post-hoc analysis in patients with type 2 diabetes mellitus on dual antidiabetes drug therapy. Nutr Diabetes. 2016;6:e217.

7. Hermanns N, Mahr M, Kulzer B, Skovlund SE, Haak T. Barriers towards insulin therapy in type 2 diabetic patients: results of an observational longitudinal study. Health Qual Life Outcomes. 2010;8:113.

8. Ali A, Cheng AYY, Yu CH. Breaking down barriers to initiating insulin Insulin prescription pad. Can Fam Physician. 2015;61:446-447.

9. Brown JB, Nichols GA, Perry A. The burden of treatment failure in type 2 diabetes. Diabetes Care. 2004,27:1535-1540.

10. Khunti K, Wolden ML, Thorsted BL, Andersen M, Davies MJ. Clinical inertia in people with type 2 diabetes: a retrospective cohort study of more than 80,000 people. Diabetes Care. 2013,36:3411-3417.

11. Harris SB, Kapor J, Lank CN, Willian AR, Houston T. Clinical inertia in patients with T2DM requiring insulin in family practice. Can Fam Physician. 2010;56:e418-e424.

12. O'Connor PJ. Improving diabetes care by combating clinical inertia. Health Serv Res. 2005;40:1854-1861.

13. Willms D, Johnson N. Essentials in qualitative research: a notebook for the field. Canada: McMaster University; 1996.
14. Krippendorff K. Content analysis: an introduction to its methodology. EE. UU.: Sage Publications Beverly Hills; 1980.

15. Smith JJ, Borgatti SP. Salience counts - and so does accuracy: correcting and updating a measure for free-list-item salience. J Linguistic Anthropol. 1998;7:208-209.

16. Thompson EC, Juan Z. Comparative cultural salience: measures using free-list data. Field Methods. 2006;18:398-412.

17. Romney AK, Weller SC, Batchelder WH. Culture as consensus: a theory of cultural and informant accuracy. Am Anthropol. 1986,88:313-338.

18. DeVellis RF. Scale development. Theory and applications. EE. UU.: Sage Publications; 1991.

19. Nunnally JC. Psychometric theory. EE. UU.: McGraw-Hill; 1978.

20. O'Connor PJ, Sperl-Hillen JA, Johnson PE, Rush WA, Biltz G. Clinical inertia and outpatient medical errors. En: Henriksen K, Battles J, Marks E, Lewin D, editores. Advances in patient safety: from research to implementation. EE. UU.: Agency for Healthcare Research and Quality; 2005.

21. Ratanawongsa N, Crosson JC, Schillinger D, Karter AJ, Saha CK, Marrero DG. Getting under the skin of clinical inertia in insulin initiation: the Translating Research Into Action for Diabetes (TRIAD) Insulin Starts Project. Diabetes Educ. 2012;38:94-100

22. Escalada J, Orozco-Beltran D, Morillas C, Alvarez-Guisasola F, Gomez-Peralta $\mathrm{F}$, Mata-Cases $\mathrm{M}$, et al. Attitudes towards insulin initiation in type 2 diabetes patients among healthcare providers: a survey research. Diabetes Res Clin Pract. 2016;122:46-53.

23. García-Morales G, Reyes-Jiménez M. Inercia clínica en pacientes con diabetes mellitus tipo 2 en una unidad de medicina familiar de Acapulco, Guerrero, México. Aten Fam. 2017;24:102-106.

24. Lerman I. Barreras que dificultan la aplicación temprana de insulina en el paciente con diabetes tipo 2. ALAD. 2009:17:66-68.

25. Alkhaifi M, Al Khusaibi GA, Theodorson T, Ward MA, Mazrou'l AA. Barriers in initiating insulin treatment in type 2 diabetes mellitus among physicians in Wilayat of Bowsher in Oman. J Fam Med Community Health. 2015;2:1034.

26. Gherman A, Veresiu IA, Sassu RA, Schnur JB, Scheckner BL, Montgomery $\mathrm{GH}$. Psychological insulin resistance: a critical review of the literature. Diabetes Int. 2011;28:125-128.

27. Lee $\mathrm{Y}$, Lee $\mathrm{P}, \mathrm{Ng}$ CJ. A qualitative study on healthcare professionals' perceived barriers to insulin initiation in a multi-ethnic population. BMC Fam Pract. 2012;13:28.

28. Owens D. Clinical evidence for the earlier initiation of insulin therapy in type 2 diabetes. Diabetes Technol Ther. 2013;15:776-785.

29. The NHS Atlas of Variation in Healthcare. Reducing unwarranted variation to increase value and improve quality. Inglaterra: Public Health England/NHS Englad/Care Quality Commission/Health and Social Care Information Centre/Office for National Statistics/NHS Improving Quality; 2016. 